\title{
Uso da estimulação transcraniana por corrente contínua em pacientes com câncer de mama com alterações cognitivas causadas pela quimioterapia: revisão integrativa
}

\author{
Renata de Lima Martins ${ }^{1,2}$ (D), Thuanne Karine do Nascimento ${ }^{1,2}$ (D), Roberto \\ Vinícius Antonino da $\operatorname{Costa}^{1}$ (D), Gabriela Brasileiro Campos Mota $^{1}$ (D) \& \\ Kedma Anne Lima Gomes ${ }^{1,3}$ (D)
}

(1) Unifacisa - Centro Universitário, Rua Manoel Cardoso Palhano 124-152, Campina Grande 58408326, Paraíba, Brasil. E-mail: thuannekarine@hotmail.com

(2) Unifacisa - Centro Universitário, Programa de Pós-Graduação em Fisioterapia em Terapia Intensiva Adulto e Infantil, Rua Manoel Cardoso Palhano 124-152, Campina Grande 58408-326, Paraíba, Brasil. E-mail: renatamarttins@outlook.com

(3) Universidade Federal da Paraíba - Campus I, Centro de Ciências Humanas, Letras e Artes, Programa de Pós-Graduação em Neurociência Cognitiva e Comportamento, Cidade Universitária, Castelo Branco 58051-900, João Pessoa, Paraíba, Brasil. E-mail: kedma.gomes@maisunifacisa.com.br

Martins R.L., Nascimento T.K., Costa R.V.A., Mota G.B.C. \& Gomes K.A.L. (2021) Uso da estimulação transcraniana por corrente contínua em pacientes com câncer de mama com alterações cognitivas causadas pela quimioterapia: revisão integrativa. Pesquisa e Ensino em Ciências Exatas e da Natureza, 5(edição especial 1): e1642. http://dx.doi.org/10.29215/pecen.v5i0.1642

Responsabilidade editorial: Patrícia Lopes Oliveira. Recebido: 02 dezembro 2020. Aceito: 07 janeiro 2021. Publicado: 04 fevereiro 2021.

Resumo: Aproximadamente $17 \%$ a $50 \%$ das pacientes com câncer de mama (CM) sofrem de comprometimento cognitivo após a conclusão do tratamento quimioterápico. Com essas manifestações negativas, inúmeros métodos vêm sendo aplicados para minimizá-las, como terapia cognitiva comportamental, treino cognitivo, treinamento de memória, programas de exercícios resistidos e caminhada. Atualmente, a Estimulação Transcraniana por Corrente Contínua (ETCC) também está sendo cogitada como possibilidade terapêutica. Este trabalho teve como objetivo verificar, com base na literatura, os efeitos da referida estimulação nos déficits cognitivos decorrentes da quimioterapia em pacientes com CM. Realizou-se uma revisão integrativa, com publicações dos últimos 10 anos nas bases de dados PubMed, Scielo, LILACS, Science Direct e Cochrane. Utilizando-se descritores em português, inglês e espanhol, indexados nas plataformas DeCS e MeSH com o auxílio do operador booleano $A N D$. Ao final, 2 artigos compuseram a amostra. Os estudos evidenciaram boa tolerância e viabilidade relacionadas ao uso da corrente, bem como melhoras nos escores dos testes de cognição global, atenção, função cognitiva autorreferida e qualidade de vida. Ao passo que apontaram caminhos metodológicos e resultados favoráveis ao uso da ETCC, suscitaram novas possibilidades para futuras pesquisas. Trata-se de um método não farmacológico, de baixo custo e seguro para a população em questão.

Palavras chave: Corrente contínua, tratamento quimioterápico, tratamento fisioterapêutico.

Use of transcranial direct current stimulation on breast cancer patients with cognitive impairment caused by chemotherapy: integrative review

Abstract: Approximately 17\% to 50\% of breast cancer patients suffer from cognitive impairment after the completion of chemotherapy. With these negative manifestations, numerous methods have been applied to minimize them, such as cognitive behavioral therapy, cognitive training, memory training, resistance exercise programs and walking. Currently, Transcranial Direct Current Stimulation (ETCC) 
is also being considered as a therapeutic possibility. This work aimed to verify, based on the literature, the effects of this stimulation on cognitive deficits resulting from chemotherapy in patients with breast cancer. An integrative review was carried out, with publications from the last 10 years in the databases PubMed, Scielo, LILACS, Science Direct and Cochrane. Using descriptors in Portuguese, English and Spanish, indexed on the DeCS and MeSH platforms with the help of the Boolean operator AND. At the end, 2 articles made up the sample. The studies showed good tolerance and feasibility related to the use of current, as well as improvements in the scores of the tests of global cognition, attention, self-reported cognitive function and quality of life. While pointing out methodological paths and results favorable to the use of ETCC, they raised new possibilities for future research. It is a non-pharmacological, low cost and safe method for the population in question.

Key-words: Direct current, chemotherapy treatment, physiotherapeutic treatment.

A quimioterapia antineoplásica utiliza agentes químicos, isolados ou em combinação, com o intuito de extinguir tumores malignos (Bonassa \& Santana 2005). Segundo Nurgali et al. (2018), os efeitos colaterais e as sequelas de longo prazo da quimioterapia anticâncer continuam sendo uma importante fonte de preocupação para pacientes e médicos, apesar da eficácia aprimorada e das chances de sobrevivência ofertadas pelos tratamentos modernos. Isto se deve ao fato de que as drogas utilizadas no tratamento quimioterápico são capazes de provocar efeitos deletérios na medula óssea, no coração e no sistema digestivo, podendo causar, ainda, comprometimentos cognitivos (Ahles 2012).

O comprometimento cognitivo relacionado ao câncer (CCRC) envolve alterações nos domínios de memória, atenção, funcionamento executivo e velocidade de processamento (Wefel et al. 2011). Estes efeitos exercem um impacto significativo na qualidade de vida, prejudicando a funcionalidade, bem como a autoestima, autoconfiança e capacidade de trabalho percebida pelos sobreviventes de câncer (Wefel et al. 2004; Reid-Arndtr et al. 2009; Von Ah et al. 2013).

Aproximadamente $17 \%$ a $50 \%$ das pacientes com câncer de mama (CM) sofrem de comprometimento cognitivo por meses ou anos após a conclusão do tratamento (Wefel et al. 2011). Um estudo comparando o desempenho cognitivo de pacientes com CM que receberam quimioterapia adjuvante com ciclofosfamida, metotrexato e fluorouracil (CMF) evidenciou que as participantes tiveram desempenho significativamente pior do que um grupo de controle saudável em testes cognitivos que avaliaram memória verbal imediata e tardia, velocidade de processamento, funcionamento executivo e velocidade psicomotora, mesmo 20 anos após o tratamento (Koppelmansk et al. 2012).

Diante destas manifestações negativas, muitos autores mencionam inúmeros métodos que vêm sendo aplicados para minimizá-las, a saber: terapia cognitiva comportamental, treino cognitivo, treinamento de memória, programas de exercícios resistidos e caminhada (Baumann et al. 2011; Ferguson et al. 2012; Schuurs \& Green 2013; Kesler et al. 2013; McDougall et al. 2014; Von Ah et al. 2013; Hartman et al. 2017).

Até o momento, sabe-se da existência de quatro estudos que pretenderam investigar a Estimulação Transcraniana por Corrente Contínua (ETCG) em pacientes com CM submetidas à quimioterapia como uma outra possibilidade terapêutica: um foi realizado por Gaynor et al. (2020) com 16 pacientes sobreviventes; outro, tratou-se de um estudo de caso conduzido por Knotkova et al. (2014) e os outros dois estão em andamento nos Estados Unidos, de forma que nenhum resultado preliminar foi publicado (Smith \& Blackwood 2017; Vannorsdall et al. 2017). A ETCC, uma corrente neuro-modulatória não invasiva que modula a função cortical, já vem sendo utilizada com o intuito de estabilizar os déficits cognitivos causados pela Doença de Alzheimer (DA), minimizando os prejuízos funcionais decorrentes da patologia (Stagg \& Nitsche 2011; Boggio et al. 2012).

Uma semelhança importante entre a fisiopatologia e os efeitos adversos da quimioterapia pode ser vista em relação ao hipocampo, que está relacionado à memória e aparece alterado em portadores de Alzheimer, ao passo que tem seu volume reduzido em pacientes com CM 
submetidas ao tratamento quimioterápico, ocorrendo mudanças nos níveis plasmáticos de Fator de Necrose Tumoral Alfa (TNF- $\alpha$ ) (Forlenza 2005; Kesler et al. 2013).

Alguns pesquisadores também vêm realizando a combinação de tarefas ou treino cognitivo durante a aplicação da ETCC como possível fator a potencializar os efeitos agudos prócognitivos, além de prolongar sua duração, de modo que já foram evidenciadas melhoras cognitivas na memória de trabalho em amostra de voluntários saudáveis com aplicação associada à execução de tarefa que recrutava o uso deste tipo de memória (Jones et al. 2015; Ruf et al. 2017).

Diante do exposto, a presente proposta de pesquisa tem sua relevância justificada com base na necessidade de verificar os efeitos de recursos não farmacológicos, de fácil aplicação e baixo custo, especificamente a ETCC, nas alterações cognitivas provocadas pelos agentes quimioterápicos, com vistas a promover melhoras nos domínios comumente afetados, otimizando a funcionalidade e a qualidade de vida de pacientes oncológicos.

Ademais, o tema consta na Agenda Nacional de Prioridades de Pesquisa em Saúde, elaborada em 2015, dentro da temática "Saúde da Mulher", que busca estabelecer ações que visem a magnitude, dinâmica e compreensão dos problemas de saúde da mulher com CM, desde o diagnóstico até a reinserção profissional desta (BRASIL 2015).

Desse modo, o estudo apresentou como objetivo verificar, com base na literatura, os efeitos da referida estimulação nos déficits cognitivos decorrentes da quimioterapia em pacientes com CM.

Tratou-se de uma revisão integrativa, a fim de verificar a aplicação da ETCC/tDCS (Transcranial direct current stimulation) como possibilidade terapêutica na melhora dos domínios cognitivos de mulheres com CM expostas a quimioterápicos.

Os seguintes descritores em português, inglês e espanhol foram escolhidos, indexados nas plataformas DeCS (Descritores em Ciências da Saúde) e MeSH (Medical Subject Headings): Cognição, neoplasias da mama, Estimulação Transcraniana por Corrente Direta/Cognition, breast neoplasms, Transcranial direct current stimulation/Cognición, neoplasias de la mama, Estimulación Transcraneal de Corriente Directa. O operador booleano AND foi utilizado. Scielo, PubMed, LILACS, Science Direct e Cochrane foram elencadas para os procedimentos.

Fizeram parte do levantamento bibliográfico os estudos publicados nos últimos 10 anos e que aplicaram a ETCC/tDCS como recurso para as alterações cognitivas presentes em pacientes com CM submetidas a quimioterápicos. Revisões e aquelas publicações que não abordavam o tema de forma satisfatória foram excluídas. Ao final, dois (02) artigos compuseram a amostra.

Seguindo a estratégia supracitada, foram encontradas duas (02) pesquisas que elegeram o uso da ETCC em pacientes com CM a fim de intervir na função cognitiva e na fadiga, sendo esses estudos finalizados. A trajetória da busca pelo ensaio clínico e estudo de caso está disposta no Quadro 1. De acordo com a amostra obtida, o Quadro 2 demonstra as informações relevantes sobre as publicações.

Os dois estudos incluídos na busca apresentaram uma média de $3.5( \pm 1.5)$ dias consecutivos dentro do protocolo de intervenção. Com exceção do estudo de caso realizado por Knotkova et al. (2014), todos os outros foram ou estão sendo conduzidos como Ensaios Clínicos, entretanto, todos os pesquisadores trouxeram a proposta de aplicar a ETCC em pacientes com CM para verificar seus efeitos nas alterações cognitivas resultantes da quimioterapia. Um estudo sugeriu o parâmetro de 2mA (Knotkova et al. 2014; Smith \& Blackwood 2017; Vannorsdall et al. 2017). Apenas Gaynor et al. (2020) ajustou o dispositivo em 1mA.

Em um ensaio clínico experimental realizado por Ballester \& Carvalho (2016) com 15 sujeitos saudáveis foi utilizado a ETCC domiciliar, em que cada sessão de estimulação anódica em córtex primário esquerdo e catódica em supraorbital direita utilizou-se uma corrente de $2 \mathrm{~mA}$ por 20 minutos. Desse modo, o desfecho primário foi a excitabilidade cortical mensurada através do parâmetro de Estimulação Magnética Transcraniana chamado de Potencial Evocado Motor (MEP). 


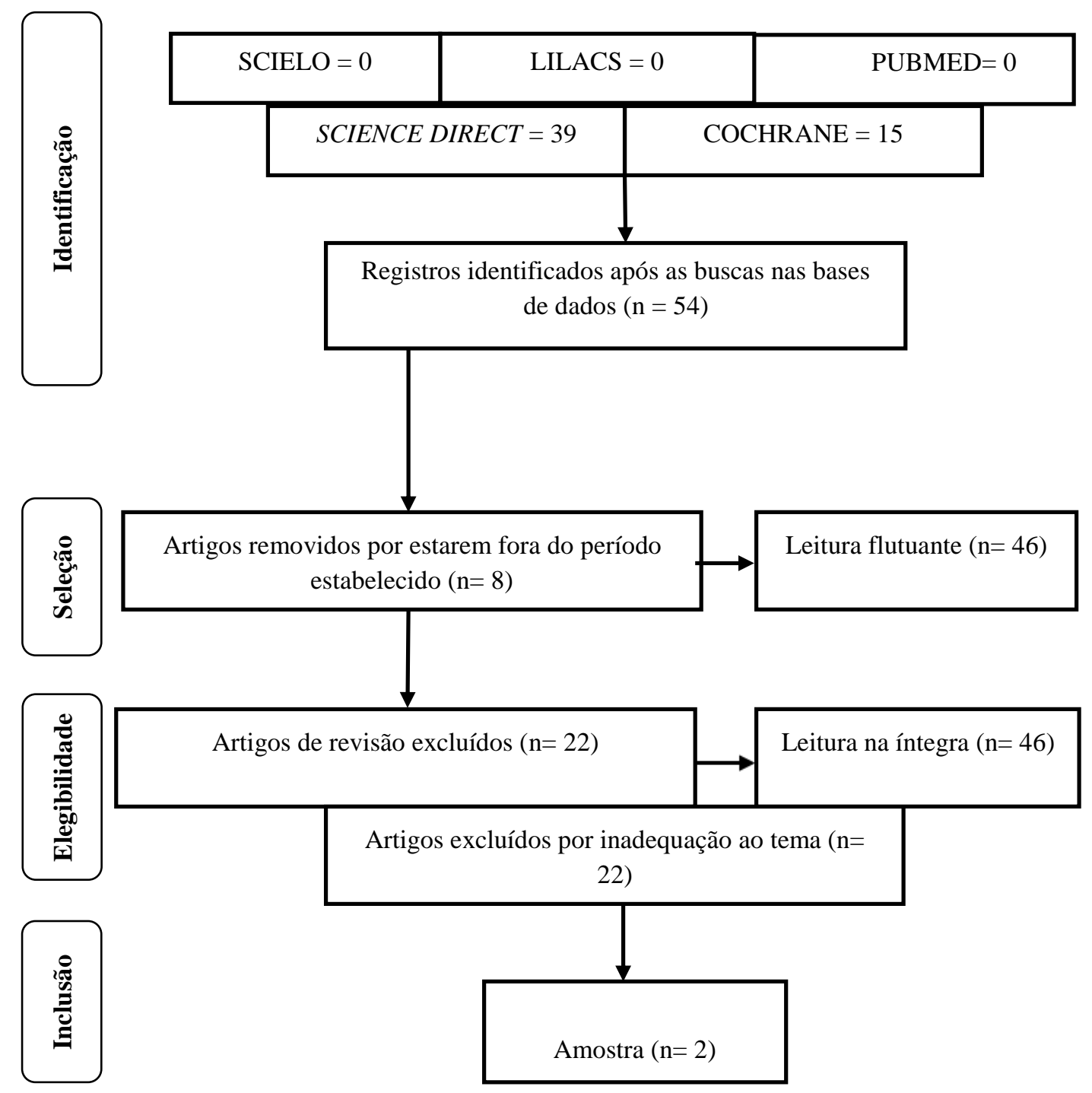

Quadro 1. Fluxograma da estratégia de busca.

No que diz respeito ao tempo de aplicação, a escolha mais frequente entre os autores foi de 30 minutos, sendo 15 minutos a menor duração da sessão entre as pesquisas. Smith \& Blackwood (2017) e Vannorsdall et al. (2017) estabeleceram ETCC placebo (Sham) para o grupo controle para fins comparativos.

Em relação ao posicionamento dos eletrodos, apenas três estudos especificaram a localização tomando como referência o sistema 10-20 da eletroencefalografia, de modo que a estimulação ativa foi destinada ao ponto F3 (córtex pré-frontal dorsolateral esquerdo - CPFDL), enquanto o ponto F4 manteve-se na configuração passiva (Knotkova et al. 2014; Smith \& Blackwood 2017; Gaynor et al. 2020).

Quanto ao momento ideal para a aplicação da ETCC, os estudos divergiram entre si: Gaynor et al. (2020) - mais de 6 meses após a conclusão do tratamento com Trastuzumabe, Docetaxel e Carboplatin, cuja administração teve duração de 34 semanas; Knotkova et al. (2014) - 8 meses após quimioterapia com Docetaxel e Carboplatin, administrada a cada 3 semanas, perfazendo um total de 52 semanas de tratamento.

As pesquisas abordaram a avaliação da cognição geral, função executiva, memória de trabalho, atenção e função conginitiva subjetiva. Além disso, alguns se dispuseram a analisar qualidade de vida, tolerância e viabilidade da ETCC. Via de regra, os autores elencaram instrumentos como Stroop Interference Tests (SIT), Immediate Recognition and Delayed 
Recognition Tests, Go-NoGo Test, Memorial Symptom Assessment Scale Short Form (MSAS-SF), Conners' Continuous Performance Test (CPT-II), Own Functioning Gating Inventory (SGI), Life Enjoyment and Satisfaction Questionnaire, Brunoni Adverse Events Questionnaire e tDCS Patient Experience Questionnaire para avaliar as variáveis supracitadas.

Quadro 2. Principais informações sobre os estudos.

\begin{tabular}{|c|c|c|c|c|c|}
\hline Autor/Ano & Título & País & $\begin{array}{l}\text { Tipo de } \\
\text { estudo }\end{array}$ & Amostra & Principais resultados \\
\hline $\begin{array}{c}\text { Knotkova et al. } \\
\text { (2014) }\end{array}$ & $\begin{array}{l}\text { Transcranial Direct } \\
\text { Current Stimulation } \\
\text { (TDCS) improved } \\
\text { cognitive outcomes in a } \\
\text { cancer survivor with } \\
\text { chemotherapy-induced } \\
\text { cognitive difficulties. }\end{array}$ & $\begin{array}{l}\text { Estados } \\
\text { Unidos }\end{array}$ & Estudo de caso & $\begin{array}{l}1 \text { mulher } \\
\text { sobrevivente de CM, } \\
\text { com } 55 \text { anos de } \\
\text { idade }\end{array}$ & $\begin{array}{l}\text { A partir do relato da } \\
\text { paciente e dos escores } \\
\text { obtidos pela avaliação } \\
\text { objetiva da cognição, } \\
\text { observou-se melhora } \\
\text { substancial na } \\
\text { memória e na função } \\
\text { executiva. }\end{array}$ \\
\hline $\begin{array}{l}\text { Gaynor et al. } \\
\quad(2020)\end{array}$ & $\begin{array}{l}\text { Impact of transcranial } \\
\text { direct current } \\
\text { stimulation on sustained } \\
\text { attention in breast } \\
\text { cancer survivors: } \\
\text { evidence for feasibility, } \\
\text { tolerability and initial } \\
\text { efficacy. }\end{array}$ & $\begin{array}{l}\text { Estados } \\
\text { Unidos }\end{array}$ & Ensaio clínico & $\begin{array}{l}16 \text { sobreviventes } \\
\mathrm{CM}\end{array}$ & $\begin{array}{l}\text { Os resultados sugerem } \\
\text { que a tDCS é viável, } \\
\text { tolerável e pode ser } \\
\text { uma intervenção eficaz } \\
\text { para melhorar as } \\
\text { dificuldades de atenção } \\
\text { sustentada em } \\
\text { sobreviventes com } \\
\text { disfunção cognitiva } \\
\text { relacionada ao câncer. }\end{array}$ \\
\hline
\end{tabular}

A quimioterapia vem sendo uma alternativa de tratamento utilizada no intuito de combate ao câncer, contudo, pesquisas em Neurociências mostram que essa vertente de tratamento pode gerar alterações de diversas naturezas, incluindo comprometimento cognitivo. Estudos anteriores mostraram que tais alterações podem estar associadas à depressão, ansiedade ou fadiga; ao passo que pesquisas realizadas recentemente apontam uma permanência dos prejuízos cognitivos, com o acometimento de domínios específicos (Lima \& Póvoa 2017).

Os dois estudos já finalizados (Knotkova et al. 2014; Gaynor et al. 2020) registraram boa tolerância e viabilidade relacionadas ao uso da ETCC, bem como melhoras nos escores dos testes de cognição global, atenção, função cognitiva autorreferida e qualidade de vida. No caso do ensaio clínico desenvolvido por Knotkova et al. (2014), esses achados foram superiores aos encontrados no grupo controle.

De acordo com Verveer et al. (2020), a ETCC é uma neuroestimulação não invasiva tolerável e não possui efeitos adversos graves conhecidos, sendo capaz de promover a modulação dos potenciais de membrana no cérebro através de pequenas correntes elétricas.

Diante da literatura analisada, foi possível concluir que a ETCC parece ser um método promissor enquanto terapia para a melhora das alterações cognitivas resultantes da quimioterapia. Estes efeitos são melhores observados com a interação da neuromodulação com outras intervenções.

Por se tratar de um método não farmacológico, de baixo custo e seguro, sugere-se que novas pesquisas sejam conduzidas para que essa possibilidade de tratamento possa ser empregada com segurança e eficiência. Ademais, é imprescindível estabelecer uma melhor padronização dos parâmetros, bem como dos locais de aplicação da corrente, de forma a contribuir para sua aplicabilidade. Por fim, acredita-se que a ETCC possa colaborar com o retorno das pacientes com CM às atividades cotidianas.

Ao passo que apontaram caminhos metodológicos e resultados favoráveis ao uso da ETCC, esses estudos suscitaram novas possibilidades para futuras pesquisas. 


\section{Referências}

Ahles T.A., Saykin A.J., McDonald B.C., Li Y., Furstenberg C.T., Hanscom B.S., Mulrooney T.J., Schwartz G.N. \& Kaufman P.A. (2010) Longitudinal assessment of cognitive changes associated with adjuvant treatment for breast cancer: impact of age and cognitive reserve. Journal of Clinical Oncology, 28(29): 4434-4440. https://doi.org/10.1200/JCO.2009.27.0827

Ballester R.F \& Carvalho F. (2016) Desenvolvimento de aparato de estimulação transcraniana de corrente contínua (ETCC) para uso domiciliar. Feira de Inovação Tecnológica da UFRGS. Porto Alegre: FINOVA.

Baumann F.T., Drosselmeyer N., Leskaroski A., Knicker A., Krakowski-Roosen H., Zopf E.M. \& Bloch W. (2011) 12-Week Resistance Training with Breast Cancer Patients during Chemotherapy: Effects on Cognitive Abilities. Breast Care, 6: 142-143. https://doi.org/10.1159/000327505

Boggio P.S., Ferrucci R., Mameli F., Martins D., Martins O., Vergari M., Tadini L., Scarpini E., Fregni F. \& Priori A. (2012) Prolonged visual memory enhancement after direct current stimulation in Alzheimer's disease. Brain Stimulation, 5(3): 223-230. https://doi.org/10.1016/j.brs.2011.06.006

Bonassa E.M.A. \& Santana T.R. (2005) Enfermagem em terapêutica oncológica. $5^{\circ}$ edição. São Paulo: Atheneu. 538 p.

BRASIL (2015) Ministério da Saúde. Agenda Nacional de Prioridades de Pesquisa em Saúde. Disponível em: http://bvsms.saude.gov.br/bvs/publicacoes/agenda_nacional_prioridades_2ed_4i mp.pdf (Acesso em 06/07/2020).

Ferguson R.J., Mcdonald B.C., Rocque M., Furstenberg C., Horrigan S., Ahles T. \& Saykin A. (2012) Development of CBT for chemotherapy-related cognitive change: Results of a waitlist control trial. Psycho-oncology, 21: 176-86. https://doi.org/10.1002/pon.1878

Forlenza O.V. (2005) Tratamento farmacológico da doença de Alzheimer. Archives of Clinical Psychiatry, 32(3): 137-148. https://doi.org/10.1590/S0101-60832005000300006

Gaynor A.M., Pergolizzi D., Alici Y., McNeal K., Ahles T.A. \& Root J.C. (2020) Impact of transcranial direct current stimulation on sustained attention in breast cancer survivors: Evidence for feasibility, tolerability, and initial efficacy. Brain Stimulation, 13(4): 1108-1116. https://doi.org/10.1016/j.brs.2020.04.013

Hartman S.J., Nelson S.H., Myers E., Natarajan L., Sears D.D., Palmer B.W., Weiner L.S., Parker B.A. \& Patterson R.E. (2017) Randomized controlled trial of increasing physical activity on objectively measured and self-reported cognitive functioning among breast cancer survivors: The memory \& motion study. Cancer, 124(1): 192-202. https://doi.org/10.1002/cncr.30987

Jones K.T., Stephens J.A., Alam M., Bikson M. \& Berryhill M.E. (2015) Longitudinal Neurostimulation in Older Adults Improves Working Memory. PLoS ONE, 10(4): e0121904. https://doi.org/10.1371/journal.pone.0121904

Kesler S.R., Watson C., Koovakkattu D., Lee C., O'Hara R., Mahaffey M.L. \& Wefel J.S. (2013) Elevated prefrontal myo-inositol and choline following breast cancer chemotherapy. Brain Imaging and Behavior, 7(4): 501-510. https://doi.org/10.1007/s11682-013-9228-1

Knotkova H., Malamud S.C. \& Cruciani R.A. (2014) Transcranial direct current stimulation (TDCS) improved cognitive outcomes in a cancer survivor with chemotherapy-induced cognitive difficulties. Brain Stimulation, 7(5): 767-768.

https://doi.org/10.1016/j.brs.2014.05.007

Koppelmans V., Breteler M.M., Boogerd W., Seynaeve C., Gundy C. \& Schagen S.B. (2012) Neuropsychological performance in survivors of breast cancer more than 20 years after adjuvant chemotherapy. Journal of Clinical Oncology, 30(10): 1080-1086. https://doi.org/0.1200/JCO.2011.37.0189

Lima C.V.C. \& Póvoa R.M.F. (2017) Mulheres Submetidas à Quimioterapia e suas Funções Cognitivas. Psicologia: Ciência e Profissão, 37(4): 970-980. https://doi.org/10.1590/1982-3703004772016 
McDougall G.J.Jr., Oliver J.S. \& Scogin F. (2014) Memory and cancer: a review of the literature. Archives of Psychiatric Nursing, 28(3): 180-186. https://doi.org/10.1016/j.apnu.2013.12.005

Nurgali K., Jagoe R.T. \& Abalo R. (2018) Editorial: Adverse Effects of Cancer Chemotherapy: Anything New to Improve Tolerance and Reduce Sequelae? Frontiers in Pharmacology, 9: 245-447. https://doi.org/10.3389/fphar.2018.00245

Reid-Arndt S.A., Yee A., Perry M.C. \& Hsieh C. (2009) Cognitive and psychological factors associated with early posttreatment functional outcomes in breast cancer survivors. Journal of Psychosocial Oncology, 27(4): 415-434. https://doi.org/10.1080/07347330903183117

Ruf S., Fallgatter A.J. \& Plewnia C. (2017) Augmentation of working memory training by transcranial direct current stimulation (tDCS). Scientific Reports, 7(2017): 876. https://doi.org/10.1038/s41598-017-01055-1

Schuurs A. \& Green H. (2013) A feasibility study of group cognitive rehabilitation for cancer survivors: Enhancing cognitive function and quality of life. Psycho-Oncology, 22(5): 10431049. https://doi.org/10.1002/pon.3102

Smith K. \& Blackwood M.M. (2017) Transcranial Direct Current Stimulation (tDCS) to Improve Fatigue and Cognitive Function. Sidney Kimmel Comprehensive Cancer Center at Johns Hopkins, ClinicalTrials.gov Identifier: NCT03487601.

Stagg C.J. \& Nitsche M.A. (2011) Physiological basis of transcranial direct current stimulation. The Neuroscientist, 17(1): 37-53. https://doi.org/10.1177/1073858410386614

Vannorsdall T.D., Smith K., Wolff A. \& Huang C.I. (2017) Reducing Breast Cancer-related Fatigue and Improving Cognition With Non-Invasive Brain Stimulation. Johns Hopkins University, ClinicalTrials.gov Identifier: NCT03143894.

Verveer I., Remmerswaal D., Jongerling J., van der Veen F.M. \& Franken I.H.A. (2020) No effect of repetitive tDCS on daily smoking behaviour in light smokers: A placebo controlled EMA study. PLOS ONE, 15(5): e0233414. https://doi.org/10.1371/journal.pone.0233414

Von Ah.D., Habermann B., Carpenter J.S. \& Schneider B.L. (2013) Impact of perceived cognitive impairment in breast cancer survivors. European Journal of Oncology Nursing, 17(2): 236241. https://doi.org/10.1016/j.ejon.2012.06.002

Wefel J.S., Vardy J., Ahles T. \& Schagen S.B. (2011) International cognition and cancer task force recommendations to harmonise studies of cognitive function in patients with cancer. The Lancet Oncology, 12(7): 703-708. https://doi.org/10.1016/S1470-2045(10)70294-1

Wefel J.S., Lenzi R., Theriault R.L., Davis R.N. \& Meyers C.A. (2004) The cognitive sequelae of standard-dose adjuvant chemotherapy in women with breast carcinoma. Cancer, 100: 22922299. https://doi:10.1002/cncr.20272 